PROCEEDINGS OF THE

AMERICAN MATHEMATICAL SOCIETY

Volume 135, Number 9, September 2007, Pages 2759-2768

S 0002-9939(07)08766-7

Article electronically published on March 30, 2007

\title{
A SCHWARZ LEMMA ON THE POLYDISK
}

\author{
GREG KNESE
}

(Communicated by Joseph A. Ball)

\begin{abstract}
We prove a generalization of the infinitesimal portion of the classical Schwarz lemma for functions from the polydisk to the disk. In particular, we describe the functions which play the role of automorphisms of the disk in this context-they turn out to be rational inner functions in the Schur-Agler class of the polydisk with an added symmetry constraint. In addition, some sufficient conditions are given for a function to be of this type.
\end{abstract}

\section{INTRODUCTION}

The Schwarz lemma on the unit disk $\mathbb{D} \subset \mathbb{C}$ says that if $f: \mathbb{D} \rightarrow \mathbb{D}$ is a holomorphic function with $f(0)=0$, then $|f(z)| \leq|z|$ for all $z \in \mathbb{D}$ and $\left|f^{\prime}(0)\right| \leq 1$. Furthermore, if equality ever occurs in either of the two preceding inequalities, then $f$ is of the form $f(z)=\lambda z$ where $|\lambda|=1$. Needless to say, this result has been generalized to many different situations.

Here we would like to focus on the infinitesimal part of the Schwarz lemma, which can be stated invariantly (without reference to the origin) as

$$
\left|f^{\prime}(z)\right| \leq \frac{1-|f(z)|^{2}}{1-|z|^{2}}
$$

provided $f$ is a holomorphic function from the disk to the disk. Equality at a single point implies equality at every point and that $f$ is an automorphism of the disk:

$$
f(z)=\lambda \frac{z-a}{1-\bar{a} z}
$$

for some unimodular constant $\lambda$ and some point $a$ in the disk. For holomorphic functions from the polydisk $\mathbb{D}^{n}=\mathbb{D} \times \cdots \times \mathbb{D}$ to the disk, we have the following well-known simple generalization (see [3], page 179).

Proposition 1.1. If $f: \mathbb{D}^{n} \rightarrow \mathbb{D}$ is holomorphic, then

$$
\sum_{i=1}^{n}\left(1-\left|z_{i}\right|^{2}\right)\left|\frac{\partial f}{\partial z_{i}}(z)\right| \leq 1-|f(z)|^{2}
$$

for any $z=\left(z_{1}, z_{2}, \ldots, z_{n}\right) \in \mathbb{D}^{n}$.

Received by the editors April 10, 2006 and, in revised form, May 1, 2006.

2000 Mathematics Subject Classification. Primary 30C80; Secondary 32A30, 47A57.

Thanks to John McCarthy for his advice at all stages of this research.

(C)2007 American Mathematical Society Reverts to public domain 28 years from publication 
In light of the situation for $n=1$, we feel compelled to ask, for which functions $f: \mathbb{D}^{n} \rightarrow \mathbb{D}$ does equality in (1.1) hold at every point? Theorem 1.2 below answers this question.

Before presenting the theorem we remind the reader of a few definitions. The set $(\partial \mathbb{D})^{n}$, which is contained in the topological boundary $\partial\left(\mathbb{D}^{n}\right)$ of the polydisk, is called the distinguished boundary of $\mathbb{D}^{n}$. Also, a holomorphic function on the polydisk is inner if its values on the distinguished boundary (which exist via radial limits almost everywhere) have modulus 1 almost everywhere. See 3 for more details on this definition.

Theorem 1.2. Let $f: \mathbb{D}^{n} \rightarrow \mathbb{D}$ be a holomorphic function which depends on all $n$ variables (i.e. none of $f$ 's partial derivatives is identically zero). Then, $f$ satisfies

$$
\sum_{i=1}^{n}\left(1-\left|z_{i}\right|^{2}\right)\left|\frac{\partial f}{\partial z_{i}}(z)\right|=1-|f(z)|^{2}
$$

for every $z \in \mathbb{D}^{n}$ if and only if there is an $(n+1) \times(n+1)$ symmetric unitary $U$ (i.e. $U^{*}=U^{-1}$ and $U=U^{t}$ ) where

$$
\left.U=\underset{\mathbb{C}}{\mathbb{C}} \begin{array}{ll}
\mathbb{C} & \mathbb{C}^{n} \\
\mathbb{C}^{n} & B \\
C & D
\end{array}\right)
$$

such that

$$
f(z)=A+B E_{z}\left(I-D E_{z}\right)^{-1} C
$$

where $E_{z}$ is the $n \times n$ diagonal matrix with diagonal entries $z_{1}, z_{2}, \ldots, z_{n}$. Such functions are rational and inner.

Interestingly, while the above extremal problem (1.2) is posed entirely within the holomorphic functions from the polydisk to the disk, its solution lies in the unit ball of the Schur-Agler class of the polydisk. Though the above functions are generalizations of automorphisms of the disk, they are obviously not automorphisms in any sense themselves. Instead, the proper way to think of them is as functions with the property that at every point of the polydisk there is a holomorphic disk passing through that point on which the restricted function is an automorphism of the disk. Put more simply, at every point of the polydisk the function is "extremal" in some direction. Let us give a name to these functions.

Definition 1.3. A holomorphic function $f: \mathbb{D}^{n} \rightarrow \mathbb{D}$ is said to be everywhere infinitesimally extremal, or extremal for short, if $f$ satisfies (1.2) at every point of $\mathbb{D}^{n}$.

The above theorem is proved using the technique commonly referred to as the "lurking isometry" technique along with the "polarization" theorem for holomorphic functions, which (loosely stated) says that if $F(z, \bar{z}) \equiv 0$, then $F(z, w) \equiv 0$. See [2] page 10 for a simple proof of this; it amounts to examining $F\left(r e^{i \theta}, r e^{-i \theta}\right)=0$ as a power series in $r$ and a Fourier series in $\theta$ (using multi-index notation).

The next obvious question is how big of a set does (1.2) need to hold in order for (1.2) to hold on the entire polydisk? A first guess might be that equality at one point implies equality at every point. The simple example $f\left(z_{1}, z_{2}\right)=\left(z_{1}+z_{2}\right) / 2$ on the bidisk proves that this is not the case. Instead, we offer the following two theorems as a substitute. 
First, we make the following definition.

Definition 1.4. Given a holomorphic function $f: \mathbb{D}^{n} \rightarrow \mathbb{D}$, we define the extreme set $X(f) \subset \mathbb{D}^{n}$ for $f$ to be the set of points for which $f$ satisfies equality in (1.2), i.e.

$$
X(f):=\left\{z \in \mathbb{D}^{n}: 1-|f(z)|^{2}=\sum_{i=1}^{n}\left(1-\left|z_{i}\right|^{2}\right)\left|\frac{\partial f}{\partial z_{i}}(z)\right|\right\} .
$$

Theorem 1.5. Let $f: \mathbb{D}^{n} \rightarrow \mathbb{D}$ be holomorphic. If $X(f)$ has nonempty interior, then $f$ is everywhere infinitesimally extremal; i.e. $X(f)=\mathbb{D}^{n}$.

The above theorem is a direct result of the proof of Theorem 1.2. In order to state the next theorem, we need another definition.

Definition 1.6. A subset $D \subset \mathbb{D}^{n}$ is called a balanced disk if $D$ is of the form

$$
D=\left\{\left(\phi_{1}(\zeta), \phi_{2}(\zeta), \ldots, \phi_{n}(\zeta)\right): \zeta \in \mathbb{D}\right\}
$$

where $\phi_{1}, \phi_{2}, \ldots, \phi_{n}$ are automorphisms of the disk.

The significance of balanced disks lies in the fact that holomorphic functions on the polydisk are maximized along certain balanced disks, just as the $\ell^{1}$ norm of a vector $v \in \mathbb{C}^{n}$ is attained by $v \cdot w$ for some $w$ with unimodular entries. The motivation for the term "balanced" is that given two points $z=\left(z_{1}, \ldots, z_{n}\right), w=$ $\left(w_{1}, \ldots, w_{n}\right)$ in a balanced disk $D \subset \mathbb{D}^{n}$ we always have that $\rho\left(z_{i}, w_{i}\right)$ does not depend on $i$, where $\rho$ is the pseudo-hyperbolic distance on the disk. It turns out that $X(f)$ is always a union of balanced disks, but as the following theorem suggests, the structure of $X(f)$ is somewhat limited.

Theorem 1.7. Let $f: \mathbb{D}^{2} \rightarrow \mathbb{D}$ be holomorphic. If $X(f)$ contains two distinct intersecting balanced disks, $f$ is everywhere infinitesimally extremal.

In section 2, we will prove Theorems 1.2 and 1.5, in section 3, we will prove Theorem 1.7 and in section 4, we will present some examples and curiosities. Specifically, we present an example (constructed using Nevanlinna-Pick interpolation on the bidisk) which shows that it is possible for $X(f)$ to contain two non-intersecting balanced disks but not be the whole bidisk. Also, we will explicitly describe the extremal functions on the bidisk which map the origin to zero. Except for the coordinate functions, the extremal functions on the bidisk never extend continuously to the closure of the bidisk. We do not know if this is the case in higher dimensions.

\section{Proofs of Theorems 1.2 and 1.5}

Proof of Theorems 1.2 and 1.5 . Let $f: \mathbb{D}^{n} \rightarrow \mathbb{D}$ be holomorphic and suppose $f$ satisfies

$$
\sum_{i=1}^{n}\left(1-\left|z_{i}\right|^{2}\right)\left|\frac{\partial f}{\partial z_{i}}(z)\right|=1-|f(z)|^{2}
$$

on some open subset $V$ of the polydisk. By assumption, $f$ depends on all $n$ variables. So, we may find a point $\alpha$ in $V$ at which none of $f$ 's partial derivatives vanish. Shrinking $V$ if necessary, we may assume $V$ is a ball on which none of $f$ 's partial 
derivatives vanishes. For $i=1,2, \ldots, n$, we can find a holomorphic square root of $\frac{\partial f}{\partial z_{i}}$ on $V$; call each square root $g_{i}$. Then, (2.1) can be rewritten as

$$
\sum_{i=1}^{n}\left(1-\left|z_{i}\right|^{2}\right)\left|g_{i}(z)\right|^{2}=1-|f(z)|^{2}
$$

for each $z \in V$. The above equation is much nicer because it allows us to polarize. Let $V^{*}$ denote the set $\{\bar{z}: z \in V\}$ and define a holomorphic function $F: V \times V^{*} \rightarrow \mathbb{C}$ by

$$
F(z, w)=(1-f(z) \overline{f(\bar{w})})-\left(\sum_{i=1}^{n}\left(1-z_{i} w_{i}\right) g_{i}(z) \overline{g_{i}(\bar{w})}\right) .
$$

Since $F(z, \bar{z})=0$ for all $z \in V$, we conclude that $F(z, w)=0$ for all $z \in V$ and $w \in V^{*}$ by polarization for holomorphic functions (see [2] page 10). Replacing $w$ with $\bar{w}$, we have that for any $z, w \in V$

$$
(1-f(z) \overline{f(w)})=\sum_{i=1}^{n}\left(1-z_{i} \bar{w}_{i}\right) g_{i}(z) \overline{g_{i}(w)},
$$

and with a little rearranging we have

$$
1+\sum_{i=1}^{n} z_{i} g_{i}(z) \bar{w}_{i} \overline{g_{i}(w)}=f(z) \overline{f(w)}+\sum_{i=1}^{n} g_{i}(z) \overline{g_{i}(w)} .
$$

The map which for each $z \in V$ sends

$$
\left(\begin{array}{c}
1 \\
z_{1} g_{1}(z) \\
\vdots \\
z_{n} g_{n}(z)
\end{array}\right) \mapsto\left(\begin{array}{c}
f(z) \\
g_{1}(z) \\
\vdots \\
g_{n}(z)
\end{array}\right)
$$

defines a unitary $U$ on the span of elements of $\mathbb{C}^{n+1}$ of the above form on the left into the span of elements of $\mathbb{C}^{n+1}$ of the above form on the right. Extending $U$ if necessary to all of $\mathbb{C}^{n+1}$ we get an $(n+1) \times(n+1)$ unitary, which we write in the following block form, in order to derive what this says about $f$ :

$$
U=\underset{\mathbb{C}^{n}}{\mathbb{C}}\left(\begin{array}{ll}
\mathbb{C} & \mathbb{C}^{n} \\
C & B
\end{array}\right) .
$$

Let $E_{z}$ be the $n \times n$ diagonal matrix with diagonal entries $z_{1}, z_{2}, \ldots, z_{n}$ and let $G(z)$ be the column vector with entries $g_{1}(z), g_{2}(z), \ldots, g_{n}(z)$. Then, (2.3) says

$$
\begin{aligned}
& A+B E_{z} G(z)=f(z), \\
& C+D E_{z} G(z)=G(z)
\end{aligned}
$$

which, first solving for $G(z)$ and then for $f(z)$, yields

$$
\begin{aligned}
G(z) & =\left(I-D E_{z}\right)^{-1} C, \\
f(z) & =A+B E_{z}\left(I-D E_{z}\right)^{-1} C
\end{aligned}
$$

for any $z \in V$. Since $V$ is an open set, equation (2.5) must therefore hold on the entire polydisk.

It still must be shown that $U$ is symmetric; i.e. $U^{t}=U$, which admittedly is a strange property for a unitary to have. Our first step will be to take the partial 
derivatives of $f$ from (2.5) and set those equal to the squares of the entries of (2.4) in order to deduce that $B^{t}=C$ and $\left(D^{t}-D\right) E_{z} G(z)=0$ for all $z \in \mathbb{D}^{n}$. Our second step will be to deduce that the set $\left\{E_{z} G(z): z \in \mathbb{D}^{n}\right\}$ spans $\mathbb{C}^{n}$, from which we may conclude $D=D^{t}$ and more importantly that $U=U^{t}$.

Let $e_{1}, e_{2}, \ldots, e_{n}$ denote the standard basis (column) vectors of $\mathbb{C}^{n}$. Then, by (2.5)

$$
\begin{aligned}
\frac{\partial f}{\partial z_{i}}(z) & =B\left(I-E_{z} D\right)^{-1} P_{i}\left(I-D E_{z}\right)^{-1} C \\
& =\left(B\left(I-E_{z} D\right)^{-1} e_{i}\right)\left(e_{i}^{t}\left(I-D E_{z}\right)^{-1} C\right) \\
& =\left(e_{i}^{t}\left(I-D^{t} E_{z}\right)^{-1} B^{t}\right)\left(e_{i}^{t}\left(I-D E_{z}\right)^{-1} C\right)
\end{aligned}
$$

where $P_{i}$ denotes orthogonal projection onto $e_{i}$ in $\mathbb{C}^{n}$. On the other hand, by (2.4)

$$
\frac{\partial f}{\partial z_{i}}(z)=\left(e_{i}^{t}\left(I-D E_{z}\right)^{-1} C\right)^{2}
$$

since $g_{i}^{2}=\frac{\partial f}{\partial z_{i}}$. Combining (2.6) and (2.7) we get

$$
\left(e_{i}^{t}\left(I-D^{t} E_{z}\right)^{-1} B^{t}\right)\left(e_{i}^{t}\left(I-D E_{z}\right)^{-1} C\right)=\left(e_{i}^{t}\left(I-D E_{z}\right)^{-1} C\right)^{2},
$$

and upon cancelling the term $e_{i}^{t}\left(I-D E_{z}\right)^{-1} C$ (which cannot be identically zero)

$$
\begin{aligned}
e_{i}^{t}\left(I-D E_{z}\right)^{-1} C & =e_{i}^{t}\left(I-D^{t} E_{z}\right)^{-1} B^{t} \text { for all } i, \text { and so } \\
\left(I-D E_{z}\right)^{-1} C & =\left(I-D^{t} E_{z}\right)^{-1} B^{t} .
\end{aligned}
$$

Setting $z=0$ we see that $B^{t}=C$. Now, multiplying (2.8) by $\left(I-D^{t} E_{z}\right)$ yields

$$
C=\left(I-D^{t} E_{z}\right)\left(I-D E_{z}\right)^{-1} C=\left(I-D E_{z}\right)^{-1} C-D^{t} E_{z}\left(I-D E_{z}\right)^{-1} C
$$

which implies

$$
D^{t} E_{z}\left(I-D E_{z}\right)^{-1} C=\left(I-D E_{z}\right)^{-1} C-C=D E_{z}\left(I-D E_{z}\right)^{-1} C
$$

which finally yields

$$
\left(D^{t}-D\right) E_{z} G(z)=0
$$

for all $z \in \mathbb{D}^{n}$.

Next, we prove that as $z$ varies over $\mathbb{D}^{n}, E_{z} G(z)$ spans all of $\mathbb{C}^{n}$. This would be extremely easy if none of $G$ 's entries were zero at the origin. For then, we could set $z=r e_{i}$, and for $r$ small enough $E_{z} G(z)$ would be a nonzero multiple of $e_{i}$. Unfortunately, it might be the case that some of $G$ 's entries vanish at the origin. We do know that none of $G$ 's entries vanish at the point $\alpha \in V$ (from way back at the beginning of the proof). So, now the strategy is to somehow shift our attention to $\alpha=\left(\alpha_{1}, \alpha_{2}, \ldots, \alpha_{n}\right)$ and try to apply a similar argument.

Let $\phi_{i}(\zeta)=\left(\alpha_{i}-\zeta\right) /\left(1-\bar{\alpha}_{i} \zeta\right)$ for $i=1,2, \ldots, n$. Using the identity

$$
1-z_{i} \bar{w}_{i}=\frac{\left(1-\phi_{i}\left(z_{i}\right) \overline{\phi_{i}\left(w_{i}\right)}\right)\left(1-\bar{\alpha}_{i} z_{i}\right)\left(1-\alpha_{i} \bar{w}_{i}\right)}{1-\left|\alpha_{i}\right|^{2}}
$$

the equation (2.2) can be rewritten as

$$
(1-f(z) \overline{f(w)})=\sum_{i=1}^{n} \frac{\left(1-\phi_{i}\left(z_{i}\right) \overline{\phi_{i}\left(w_{i}\right)}\right)\left(1-\bar{\alpha}_{i} z_{i}\right)\left(1-\alpha_{i} \bar{w}_{i}\right)}{1-\left|\alpha_{i}\right|^{2}} g_{i}(z) \overline{g_{i}(w)},
$$


and we get a new $(n+1) \times(n+1)$ unitary $\tilde{U}$ which maps

$$
\left(\begin{array}{c}
1 \\
\frac{\alpha_{1}-z_{1}}{\sqrt{1-\left|\alpha_{1}\right|^{2}}} g_{1}(z) \\
\vdots \\
\frac{\alpha_{n}-z_{n}}{\sqrt{1-\left|\alpha_{n}\right|^{2}}} g_{n}(z)
\end{array}\right) \mapsto\left(\begin{array}{c}
f(z) \\
\frac{1-\overline{\alpha_{1}} z_{1}}{\sqrt{1-\left|\alpha_{1}\right|^{2}}} g_{1}(z) \\
\vdots \\
\frac{1-\bar{\alpha}_{n} z_{n}}{\sqrt{1-\left|\alpha_{n}\right|^{2}}} g_{n}(z)
\end{array}\right) .
$$

This unitary is, in fact, uniquely determined because, looking at the left hand side, and setting in turn $z=\alpha, \alpha+r e_{1}, \ldots, \alpha+r e_{n}$ for $r$ small enough, we see that the left hand side spans all of $\mathbb{C}^{n+1}$ as $z$ varies over the polydisk since $g_{i}(\alpha) \neq 0$ for $i=1,2, \ldots, n$. Therefore, the vectors on the right hand side of (2.9) span all of $\mathbb{C}^{n+1}$. This implies that vectors of the form

$$
\left(\begin{array}{c}
f(z) \\
\left(1-\bar{\alpha}_{1} z_{1}\right) g_{1}(z) \\
\vdots \\
\left(1-\bar{\alpha}_{n} z_{n}\right) g_{n}(z)
\end{array}\right)
$$

span $\mathbb{C}^{n+1}$. But, a vector of the above form is the image of

$$
\left(\begin{array}{c}
1 \\
z_{1} g_{1}(z) \\
\vdots \\
z_{n} g_{n}(z)
\end{array}\right)
$$

under the invertible matrix $U-T$, where $T$ is the diagonal matrix with diagonal $0, \bar{\alpha}_{1}, \bar{\alpha}_{2}, \ldots, \bar{\alpha}_{n}$. Hence, vectors of the form (2.10) span $\mathbb{C}^{n+1}$, and consequently, vectors of the form $E_{z} G(z)$ span $\mathbb{C}^{n}$, as desired.

This establishes the fact that $U=U^{t}$.

Conversely, suppose $f$ is of the form (2.5) for some symmetric unitary. It should be noted that $f$ does indeed map into the disk, because of the formula

$$
1-|f(z)|^{2}=C^{*}\left(I-E_{z}^{*} D^{*}\right)^{-1}\left(I-E_{z}^{*} E_{z}\right)\left(I-D E_{z}\right)^{-1} C
$$

which holds regardless of whether or not $U$ is symmetric. But, assuming $U$ is symmetric, the above simplifies to

$$
1-|f(z)|^{2}=\sum_{i=1}^{n}\left(1-\left|z_{i}\right|^{2}\right)\left|e_{i}^{t}\left(I-D E_{z}\right)^{-1} C\right|^{2} .
$$

Also, since $U$ is symmetric, the right hand side is equal to

$$
\sum_{i=1}^{n}\left(1-\left|z_{i}\right|^{2}\right)\left|\frac{\partial f}{\partial z_{i}}(z)\right|
$$

by (2.6), which was derived straight from (2.5).

Finally, $f$ 's of the form (2.5) are rational of degree at most $2 n$ by Cramer's rule. They are inner by (2.11) because the nonidentically zero polynomial $\operatorname{det}\left(I-D E_{z}\right)$ cannot vanish on a set of positive measure on the distinguished boundary of $\mathbb{D}^{n}$.

We have also established Theorem 1.5. because we proved that if (2.1) holds on an open set, then $f$ is of the form (1.3), which in turn implies that (1.2) holds everywhere. 
In closing the proof, we remark that the condition that $f$ depends on all $n$ variables is simply there to make the theorem statement nicer. If $f$ doesn't depend on some variable, then $f$ can be treated as a function on a lower dimensional polydisk.

\section{Proof of Theorem 1.7}

For the rest of the paper we will restrict our attention to the case $n=2$; we will write points of $\mathbb{D}^{2}$ as $(z, w)$, and we will write partial derivatives of $f$ as $f_{1}$ and $f_{2}$. We recall the following definitions from the introduction, restricted to the case $n=2$.

Definition 3.1. Let $f: \mathbb{D}^{2} \rightarrow \mathbb{D}$ be holomorphic. Then, the extreme set of $f$ is the subset of $\mathbb{D}^{2}$ defined by

$X(f):=\left\{(z, w) \in \mathbb{D}^{2}: 1-|f(z, w)|^{2}=\left(1-|z|^{2}\right)\left|f_{1}(z, w)\right|+\left(1-|w|^{2}\right)\left|f_{2}(z, w)\right|\right\}$.

Definition 3.2. A subset $D \subset \mathbb{D}^{2}$ is called a balanced disk if $D$ is of the form

$$
D=\left\{\left(\phi_{1}(\zeta), \phi_{2}(\zeta)\right): \zeta \in \mathbb{D}\right\}
$$

where $\phi_{1}$ and $\phi_{2}$ are automorphisms of the disk.

Observe that two balanced disks intersect at no points, one point, or all points.

Lemma 3.3. Let $f: \mathbb{D}^{2} \rightarrow \mathbb{D}$ be holomorphic. If either of $f$ 's partial derivatives equals zero at a point of $X(f)$, then $f$ depends on only one variable and $X(f)=\mathbb{D}^{2}$. Furthermore, if $f$ depends on both variables, then through each point $P$ of $X(f)$ there is a unique balanced disk $D$ contained in $X(f)$ and containing $P$ such that the restriction of $f$ to $D$ is an automorphism of the disk.

Here, $f$ restricted to a disk $D=\left(\phi_{1}, \phi_{2}\right)(\mathbb{D})$ (where $\phi_{1}$ and $\phi_{2}$ are automorphisms of the unit disk) being an automorphism of the unit disk just means $f \circ\left(\phi_{1}, \phi_{2}\right)$ is an automorphism.

Proof. It is a simple calculation to verify that if $P \in X(f), \phi$ is an automorphism of the disk, and $\Phi$ is an automorphism of the bidisk, then $\Phi^{-1}(P) \in X(\phi \circ f \circ \Phi)$. Therefore, to prove the first part of the lemma, it is sufficient to prove $f(z, w)=w$ under the assumptions $(0,0) \in X(f), f(0,0)=0, f_{1}(0,0)=0$ and $f_{2}(0,0)=1$ by applying appropriate automorphisms. For any $(z, w) \in \mathbb{D}^{2}$ with $|w|>|z|$ we see that the function from the disk to the disk defined by $g(\zeta)=f((z / w) \zeta, \zeta)$ satisfies $g^{\prime}(0)=1$ and $g(0)=0$. By the classical Schwarz lemma, $g(\zeta)=\zeta$ for all $\zeta \in \mathbb{D}$ and in particular $f(z, w)=w$. It follows that this holds on the entire bidisk.

By the first part of the lemma, if $f$ depends on both variables, then neither of $f$ 's partial derivatives can vanish at a point of $X(f)$. To prove the second portion of the lemma, we can assume like before that $P=(0,0), f(0,0)=0, f_{1}(0,0)=a$, and $f_{2}(0,0)=b$ where $a$ and $b$ are positive real numbers with $a+b=1$. A balanced disk through the origin can be written in the form $\{(\zeta, \mu \zeta): \zeta \in \mathbb{D}\}$ where $|\mu|=1$. Now, $h(\zeta)=f(\zeta, \mu \zeta)$ is an automorphism of the disk precisely when $|a+\mu b|=1$ or equivalently when $\mu=1$. Therefore, $D=\{(\zeta, \zeta)\}$ is the unique balanced disk containing $P$ on which $f$ is an automorphism of the disk. Finally, $D \subset X(f)$ because if $h(\zeta)=f(\zeta, \zeta)=\zeta$, then

$$
1=\left|h^{\prime}(\zeta)\right|=\left|f_{1}(\zeta, \zeta)+f_{2}(\zeta, \zeta)\right| \leq\left|f_{1}(\zeta, \zeta)\right|+\left|f_{2}(\zeta, \zeta)\right| \leq 1
$$


which implies $(\zeta, \zeta) \in X(f)$ for all $\zeta \in \mathbb{D}$.

In order to prove Theorem 1.7 we need to give a name to a special class of balanced disks associated to $f$.

Definition 3.4. A balanced disk $D$ is called an extreme disk for $f$ if $f$ restricted to $D$ is an automorphism of the unit disk $\mathbb{D}$.

A result of Lemma 3.3 is the following: if $f: \mathbb{D}^{2} \rightarrow \mathbb{D}$ has two intersecting extreme disks, then $X(f)=\mathbb{D}^{2}$. We are now ready to prove Theorem 1.7 .

Proof of Theorem 1.7. We may assume that the intersection point is the origin and the two disks are $D_{1}=\{(\zeta, \zeta): \zeta \in \mathbb{D}\}$ and $D_{2}=\{(\zeta, \mu \zeta): \zeta \in \mathbb{D}\}$ where $|\mu|=1$. We may also assume $D_{1}$ is an extreme disk, because $f$ has an extreme disk through the origin, and we might as well assume $D_{1}$ is it. Also, assuming $f$ depends on both variables, which we do, this implies $D_{2}$ is not an extreme disk.

Now, let $P=(a, b)$ be some point of $X(f)$. We can actually derive a formula for the extreme disk passing through $P$. Let $\phi_{c}: \mathbb{D} \rightarrow \mathbb{D}$ be the automorphism of the disk for which $0 \mapsto c, c \mapsto 0$, and $\phi_{c}=\phi_{c}^{-1}$; i.e.

$$
\phi_{c}(\zeta)=\frac{c-\zeta}{1-\bar{c} \zeta}
$$

Then, the derivative of $g(\zeta)=f\left(\phi_{a}(\zeta), \phi_{b}(\lambda \zeta)\right)$ at 0 satisfies

$$
\begin{aligned}
\left|g^{\prime}(0)\right| & =\left|f_{1}(P)\left(1-|a|^{2}\right)+\lambda f_{2}(P)\left(1-|b|^{2}\right)\right| \\
& =\left|f_{1}(P)\right|\left(1-|a|^{2}\right)+\left|f_{2}(P)\right|\left(1-|b|^{2}\right) \\
& =1-|g(0)|^{2}
\end{aligned}
$$

exactly when $\lambda$ satisfies

$$
\lambda=\operatorname{sgn}\left[\frac{f_{1}(P)}{f_{2}(P)}\right]
$$

(which makes sense because neither partial derivatives can vanish at a point of $X(f)$ in the bidisk without vanishing everywhere), in which case $g$ is an automorphism of the disk and

$$
D(P)=\left\{\left(\phi_{a}(\zeta), \phi_{b}(\lambda \zeta)\right): \zeta \in \mathbb{D}\right\}
$$

is the extreme disk passing through $P$. So, defining $\lambda(P)$ for each $P \in X(f)$ as in (3.2), we can define a map $F$ from $\mathbb{D}^{2}$ into $X(f)$ by mapping

$$
(z, w) \mapsto\left(\phi_{z}(w), \phi_{\mu z}(\lambda(z, \mu z) w)\right.
$$

The map $F$ is injective for the following reasons. If $F(z, w)=F\left(z^{\prime}, w^{\prime}\right)$, then the extreme disk through $(z, \mu z)$ intersects the extreme disk through $\left(z^{\prime}, \mu z^{\prime}\right)$. These two extreme disks must therefore be the same, since we are assuming $f$ depends on both variables. This implies $z=z^{\prime}$, because otherwise $D_{2}$ would contain two points of the same extreme disk, and since $D_{2}$ is balanced this would force $D_{2}$ to be an extreme disk, which it is not. This immediately gives $w=w^{\prime}$. Since $F$ is continuous (or even smooth), we see that $X(f)$ has nonempty interior and therefore $X(f)=\mathbb{D}^{2}$. 


\section{EXAMPLES AND CURIOSITIES}

4.1. Example: A function with two non-intersecting extreme disks. Define $g: \mathbb{D}^{2} \rightarrow \mathbb{D}$ by

$$
\begin{aligned}
g(z, w)= & \left\{(z+w) / 2+(\sqrt{5} / 6)\left(z^{2}-w^{2}\right)-(1 / 9)\left(z^{2}+7 z w+w^{2}\right)\right. \\
& \left.-(\sqrt{5} / 3)\left(z^{2} w-z w^{2}\right)-(1 / 2)\left(z^{2} w+z w^{2}\right)+z^{2} w^{2}\right\} \\
\div & \left\{1-(1 / 2)(z+w)+(\sqrt{5} / 3)(z-w)-(1 / 9)\left(z^{2}+7 z w+w^{2}\right)\right. \\
& \left.-(\sqrt{5} / 6)\left(z^{2}-w^{2}\right)+(1 / 2)\left(z^{2} w+z w^{2}\right)\right\} .
\end{aligned}
$$

This function $g$ has two non-intersecting extreme disks since

$$
\begin{gathered}
g(z, z)=z, \\
g\left(\frac{z-\sqrt{5} / 3}{1-(\sqrt{5} / 3) z}, \frac{z+\sqrt{5} / 3}{1+(\sqrt{5} / 3) z}\right)=z
\end{gathered}
$$

and

$$
\frac{z-\sqrt{5} / 3}{1-(\sqrt{5} / 3) z} \neq \frac{z+\sqrt{5} / 3}{1+(\sqrt{5} / 3) z} \text { for all } z \in \mathbb{D} \text {. }
$$

However, $g$ does not satisfy (1.2) everywhere.

The function $g$ was constructed using the Nevanlinna-Pick interpolation theorem on the bidisk by looking for solutions to the four point problem

$$
\begin{aligned}
& (0,0) \mapsto 0, \\
& (r, r) \mapsto r, \\
& (0, b) \mapsto \alpha, \\
& (a, 0) \mapsto \beta
\end{aligned}
$$

where $r<1$ and $|b|=|a|=|(\alpha-\beta) /(1-\bar{\alpha} \beta)|<1$. (This is just a pair of extremal two-point problems.) For the statement of the appropriate theorem see [1] chapter 11.

4.2. Everywhere infinitesimally extremal functions on the bidisk. Using Theorem 1.2 it is a relatively simple task to write down explicitly the everywhere infinitesimally extremal functions on the bidisk which map the origin to zero. They are of the following form:

$$
f(z, w)=\mu \frac{a z+b w-z w}{1-\bar{b} z-\bar{a} w}
$$

where $|\mu|=|a|+|b|=1$. Notice that unless $a$ or $b$ equals zero, $f$ never extends continuously to the closed bidisk. Indeed, supposing $a$ and $b$ are both positive real numbers and $\mu=1$ (after composing $f$ with an automorphism), we see that

$$
\lim _{\theta \rightarrow 0} f\left(e^{i \theta}, e^{i t \theta}\right)= \begin{cases}1 & \text { if } t \neq-b / a \\ -1 & \text { if } t=-b / a\end{cases}
$$

This immediately implies that on the bidisk the only extremal functions that extend continuously to the closed bidisk depend on one variable and are simply automorphisms of the disk (in one variable); i.e. $f(z, w)=\phi(z)$ or $f(z, w)=\phi(w)$ for some automorphism of the disk, $\phi$. 


\section{REFERENCES}

1. J. Agler and J.E. McCarthy. Pick Interpolation and Hilbert Function Spaces. American Mathematical Society, Providence, 2002. MR.1882259 (2003b:47001)

2. J.P. D'Angelo. Several Complex Variables and the Geometry of Real Hypersurfaces. CRC, Boca Raton, FL, 1993. MR 1224231 (94i:32022)

3. W. Rudin Function Theory in Polydiscs. Benjamin, New York, 1969. MR0255841 (41:501)

Department of Mathematics, Washington University in St. Louis, St. Louis, Missouri 63130

E-mail address: geknese@math.wustl.edu

$U R L:$ http://www. math. wustl.edu/ geknese 